\title{
IMPROVED V II $\log (g f)$ VALUES, HYPERFINE STRUCTURE CONSTANTS, AND ABUNDANCE DETERMINATIONS IN THE PHOTOSPHERES OF THE SUN AND METAL-POOR STAR HD 84937
}

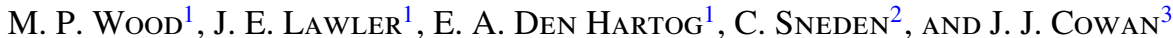 \\ ${ }^{1}$ Department of Physics, University of Wisconsin, Madison, WI 53706, USA; mpwood@wisc.edu, jelawler@wisc.edu, eadenhar@wisc.edu \\ ${ }^{2}$ Department of Astronomy and McDonald Observatory, University of Texas, Austin, TX 78712, USA; chris@ verdi.as.utexas.edu \\ ${ }^{3}$ Homer L. Dodge Department of Physics and Astronomy, University of Oklahoma, Norman, OK 73019, USA; cowan@nhn.ou.edu \\ Received 2014 July 9; accepted 2014 August 7; published 2014 September 18
}

\begin{abstract}
New experimental absolute atomic transition probabilities are reported for 203 lines of V II. Branching fractions are measured from spectra recorded using a Fourier transform spectrometer and an echelle spectrometer. The branching fractions are normalized with radiative lifetime measurements to determine the new transition probabilities. Generally good agreement is found between this work and previously reported V II transition probabilities. Two spectrometers, independent radiometric calibration methods, and independent data analysis routines enable a reduction in systematic uncertainties, in particular those due to optical depth errors. In addition, new hyperfine structure constants are measured for selected levels by least squares fitting line profiles in the FTS spectra. The new V II data are applied to high resolution visible and UV spectra of the Sun and metal-poor star HD 84937 to determine new, more accurate $\mathrm{V}$ abundances. Lines covering a range of wavelength and excitation potential are used to search for non-LTE effects. Very good agreement is found between our new solar photospheric $\mathrm{V}$ abundance, $\log \varepsilon(\mathrm{V})=3.95$ from $15 \mathrm{~V}$ II lines, and the solar-system meteoritic value. In $\mathrm{HD} 84937$, we derive $[\mathrm{V} / \mathrm{H}]=-2.08$ from 68 lines, leading to a value of $[\mathrm{V} / \mathrm{Fe}]=0.24$
\end{abstract}

Key words: atomic data - methods: laboratory: atomic - stars: abundances - stars: individual (HD 84937) Sun: abundances

Online-only material: color figures, machine-readable tables

\section{INTRODUCTION}

Stellar abundances, particularly their trends as a function of metallicity, are tests for models of nucelosynthesis and provide valuable information regarding the evolution of chemical elements in the Galaxy. Abundances in old, metal-poor stars are especially valuable since they represent the "fossil-record" of nucleosynthesis in the earliest generations of stars. Studies of metal-poor stars have found large and unexpected iron (Fe)-group abundance trends (McWilliam et al. 1995a, 1995b; McWilliam 1997; Westin et al. 2000; Cowan et al. 2002; Sneden et al. 2003; Cayrel et al. 2004; Barklem et al. 2005; Lai et al. 2008; Bonifacio et al. 2009; Roederer 2009; Suda et al. 2011; Yong et al. 2013) which have thus far refused explanation using current models of early Galactic supernova yields. These relative Fe-group abundance trends can cover \pm 1 dex for metallicities ranging from solar $([\mathrm{Fe} / \mathrm{H}] \equiv 0)$ to -4 (e.g., Figure 12 of McWilliam 1997). ${ }^{4}$

It may be that these unexpected trends represent a failure for models of nucleosynthesis in the early Galaxy. However, these trends may also indicate the breakdown of standard abundance derivation techniques in low metallicity stars, or they may result from inaccurate laboratory atomic data. Fairly comprehensive databases of atomic transition probabilities exist (e.g., NIST Atomic Spectra Database ${ }^{5}$ and Vienna Atomic Line Database ${ }^{6}$. These are of utmost importance for stellar abundance determinations. To obtain the most accurate abundances it is crucial to

\footnotetext{
4 We adopt standard spectroscopic notations. For elements $\mathrm{X}$ and $\mathrm{Y}$, the relative abundances are written $[\mathrm{X} / \mathrm{Y}]=\log _{10}\left(N_{\mathrm{X}} / N_{\mathrm{Y}}\right)_{\mathrm{star}}-\log _{10}\left(N_{\mathrm{X}} / N_{\mathrm{Y}}\right)_{\odot}$. For element $\mathrm{X}$, the "absolute" abundance is written $\log \varepsilon(\mathrm{X})=$ $\log _{10}\left(N_{\mathrm{X}} / N_{\mathrm{H}}\right)+12$. Metallicity is defined as the $[\mathrm{Fe} / \mathrm{H}]$ value.

5 Available at http://www.nist.gov/pml/data/asd.cfm.

6 Available at http://www.astro.uu.se/ vald/php/vald.php.
}

use lines that are unsaturated in the photosphere of the star being investigated. For studies covering a wide metallicity range, this requirement necessitates the use of many lines covering a range of excitation potential (E.P.) and $\log (g f)$ values, which introduces the possibility that inaccurate laboratory atomic data are affecting the measured abundances. In the photospheres of stars of interest, neutral atoms (first spectra) are a minor ionization stage while singly ionized atoms (second spectra) represent the dominant ionization stage. Therefore, in order to avoid saturation in higher metallicity stars, one makes use of weak first spectra lines that arise from high E.P. levels. In lower metallicity stars, however, one must change to stronger lines with lower E.P. values, and assuming suitable second spectra lines exist in the observed spectra, singly ionized lines become important as well. The strength of these high and low E.P. lines can vary by orders of magnitude, making it rather difficult to measure both with small uncertainties in the same laboratory spectra. If the laboratory atomic data are not at fault, the unexpected Fe-group abundance trends might result from the failure of 1D/LTE (one-dimensional/local thermodynamic equilibrium) approximations traditionally incorporated into photospheric models used for abundance determinations in metal-poor stars of interest (e.g., Asplund 2005). Giant stars are favored in studies of metal-poor stars to provide large photon fluxes for high signal-to-noise $(\mathrm{S} / \mathrm{N})$, high-resolution spectra. The combination of low-density atmospheres and reduced electron pressure from a lower metal content leads to lower collision rates in metalpoor giant stars, which may result in departures from LTE. The two possible explanations described above for the unexpected trends can be investigated with expanded and more accurate experimental Fe-group transition probabilities. One approach to determine if the trends result from $3 \mathrm{D} /$ non-LTE effects is to search for anomalous abundance measurements from various 
Table 1

Fourier Transform Spectra of a Custom Water-Cooled V Hollow Cathode Discharge (HCD) Lamp

\begin{tabular}{|c|c|c|c|c|c|c|c|c|c|c|}
\hline Index & Date & $\begin{array}{c}\text { Serial } \\
\text { Number }\end{array}$ & $\begin{array}{c}\text { Buffer } \\
\text { Gas }\end{array}$ & $\begin{array}{c}\text { Lamp } \\
\text { Current } \\
(\mathrm{mA})\end{array}$ & $\begin{array}{c}\text { Wavenumber } \\
\text { Range } \\
\left(\mathrm{cm}^{-1}\right)\end{array}$ & $\begin{array}{l}\text { Limit of } \\
\text { Resolution } \\
\left(\mathrm{cm}^{-1}\right)\end{array}$ & Coadds & $\begin{array}{l}\text { Beam } \\
\text { Splitter }\end{array}$ & Filter & Detector $^{\mathrm{a}}$ \\
\hline 1 & 1984 Dec. 9 & 3 & $\mathrm{Ar}-\mathrm{Ne}$ & 600 & $7764-49105$ & 0.057 & 12 & UV & & Mid Range Si Diode \\
\hline 2 & 1984 Dec. 9 & 4 & Ar-Ne & 300 & $7764-49105$ & 0.057 & 8 & UV & & Mid Range Si Diode \\
\hline 3 & 1984 Dec. 9 & 5 & Ar-Ne & 150 & $7764-49105$ & 0.057 & 8 & UV & & Mid Range Si Diode \\
\hline 4 & 1986 July 30 & 9 & $\mathrm{Ar}$ & 500 & $14924-37018$ & 0.048 & 4 & UV & $\mathrm{CuSO}_{4}$ & Large UV Si Diode \\
\hline 5 & 1986 July 30 & 10 & $\mathrm{Ar}$ & 500 & $14924-37018$ & 0.048 & 4 & UV & $\mathrm{CuSO}_{4}$ & Large UV Si Diode \\
\hline 6 & 1981 June 16 & 7 & Ar & 332 & $6924-37564$ & 0.043 & 8 & UV & WG295 & UV Mid Range Si Diode \\
\hline 7 & 1981 June 15 & 3 & $\mathrm{Ar}$ & 250 & $14878-36533$ & 0.043 & 8 & UV & $\mathrm{CuSO}_{4} \mathrm{WG}_{295}$ & UV Mid Range Si Diode \\
\hline 8 & 1979 Dec. 12 & 9 & $\mathrm{Ar}$ & 300 & $12422-31054$ & 0.042 & 10 & UV & TC+ 4-97 WG345 & Mid Range Si Diode \\
\hline 9 & 1979 Dec. 12 & 8 & $\mathrm{Ar}$ & 300 & $7716-22421$ & 0.030 & 8 & UV & GG945 & Super Blue Si Diode \\
\hline 10 & 1980 Sept. 4 & 1 & $\mathrm{Ar}$ & 110 & $0-17837$ & 0.023 & 5 & UV & RG610 & $\mathrm{InSb}$ \\
\hline 11 & 1983 Nov. 30 & 3 & $\mathrm{Ar}$ & 460 & 2799-9518 & 0.011 & 17 & $\mathrm{CaF}_{2}$ & $\mathrm{Si}$ & $\mathrm{InSb}$ \\
\hline 12 & 1983 Apr. 17 & 4 & $\mathrm{Ar}-\mathrm{Ne}$ & 370 & $1534-5769$ & 0.011 & 80 & $\mathrm{CaF}_{2}$ & $\mathrm{Ge}$ & $\mathrm{InSb}$ \\
\hline 13 & 1984 July 25 & 5 & $\mathrm{Ne}$ & 1000 & $12948-45407$ & 0.054 & 8 & UV & $\mathrm{CuSO}_{4}$ & $\begin{array}{l}\text { R166 photomultiplier } \\
\text { Mid Range Si Diode }\end{array}$ \\
\hline
\end{tabular}

Notes. All spectra were recorded using the $1.0 \mathrm{~m}$ FTS on the McMath telescope at the National Solar Observatory, Kitt Peak, AZ.

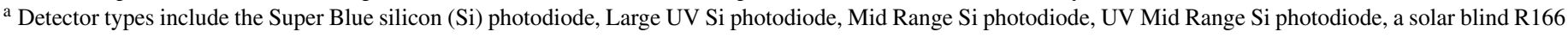
photomultiplier, and InSb detectors for the IR. The UV beam splitter is fused silica.

lines covering a range of E.P., $\log (g f)$, and wavelength for a wide range of stellar types. If improved atomic data and targeted searches for 3D/non-LTE effects fail to eliminate the observed Fe-group abundance trends, it would provide evidence that nucleosynthetic models for the early Galaxy are incomplete and need to be reexamined.

Our group has an effort underway to expand sets of transition probabilities and reduce transition probability uncertainties for Fe-group lines. Den Hartog et al. (2011) focuses on selected multiplets in Mn I and Mn II that cover small wavelength ranges and/or are Russell Saunders (LS) multiplets. Given these benefits, it is possible to reduce the Mn transition probability uncertainties to 0.02 dex with $2 \sigma$ confidence. Such small uncertainties are practical only under favorable conditions and in general are difficult to achieve. A broader approach is taken in the recent work on Ti I (Lawler et al. 2013), Ti II (Wood et al. 2013), and Ni I (Wood et al. 2014) by attempting measurements on every possible line connecting to upper levels with previously reported radiative lifetimes. This results in a larger set of transition probability measurements, though often with higher uncertainties $(0.02$ to $\sim 0.10 \mathrm{dex})$. However, small $(\sim 0.02 \mathrm{dex})$ uncertainties, such as those reported by Den Hartog et al. (2011), are not necessary for the detection of non-LTE effects in metalpoor stars. Mn I resonance lines connected to the ground level show non-LTE effects of 0.5-1 dex (J. S. Sobeck et al. 2014, in preparation).

The V II study reported herein follows the broad approach previously used for Ti I, Ti II, and Ni I by attempting transition probability measurements for all lines connected to the 31 odd-parity upper levels with laser induced fluorescence (LIF) lifetime measurements by Den Hartog et al. (2014), plus an additional odd-parity upper level with a previously reported lifetime (Biémont et al. 1989; Xu et al 2006). The result is a set of 203 transition probabilities covering a wide range of E.P., $\log (g f)$, and wavelength. Uncertainties range from $0.02 \mathrm{dex}$ for dominant branches, primarily determined by the LIF lifetime uncertainties, to $\sim 0.12$ dex for weak branches widely separated in wavelength from the dominant branch(es). The uncertainties on weak branches, which are often the most important for accurate abundance measurements, primarily result from $\mathrm{S} / \mathrm{N}$ effects, uncertainty in the radiometric calibration, or both. The use of both a Fourier transform spectrometer (FTS) and an echelle spectrometer, with independent radiometric calibrations, serves to reduce systematic uncertainties on weak line measurements.

In Sections 2 and 3 we describe the spectra recorded using the FTS and echelle spectrometer, and in Section 4 we discuss the determination of V II branching fractions from these spectra. In Section 5 we present new absolute transition probabilities with comparisons to previous results in the literature. In Section 6 new hyperfine structure (HFS) constants and component patterns derived from our FTS spectra are introduced. We apply the new V II data to determine the photospheric V abundance of the Sun in Section 7 and metal-poor star HD 84937 in Section 8.

\section{FOURIER TRANSFORM SPECTROMETER DATA}

This V II transition probability study makes use of archived FTS data from the $1.0 \mathrm{~m}$ FTS previously at the National Solar Observatory (NSO) on Kitt Peak. The NSO 1.0 m FTS has a large etendue (like all interferometric spectrometers), a resolution limit as small as $0.01 \mathrm{~cm}^{-1}$, wavenumber accuracy to 1 part in $10^{8}$, broad spectral coverage from the near ultraviolet (UV) to infrared (IR), and a high data collection rate (Brault 1976). Unfortunately the NSO FTS has been dismantled, and while there are plans to restore it to full operational status at a university laboratory, it is currently unavailable to guest observers. Table 1 lists the 13 FTS spectra used in this V II branching fraction study. All spectra, raw interferograms, and header files are available in the NSO electronic archives. ${ }^{7}$

Multiple FTS spectra are needed to determine high-quality branching fractions. Optimum sensitivity is achieved for different spectral ranges using various beam splitter, filter, and detector combinations. Although branching fractions are determined mainly from spectra obtained with lamps having an $\mathrm{Ar}$ gas fill, additional spectra are needed from lamps with a $\mathrm{Ne}$ buffer gas to allow for the correction of blends between $\mathrm{V}$ and

\footnotetext{
7 Available at http://nsokp.nso.edu/.
} 
Table 2

Echelle Spectra of Commercial V HCD Lamps

\begin{tabular}{|c|c|c|c|c|c|c|c|c|}
\hline Index & Date & Serial Numbers $^{\mathrm{a}}$ & Buffer Gas & $\begin{array}{c}\text { Lamp Current } \\
(\mathrm{mA})\end{array}$ & $\begin{array}{c}\text { Wavelength Range } \\
(\AA)\end{array}$ & Resolving Power & Coadds & $\begin{array}{c}\text { Exposure Time } \\
\text { (s) }\end{array}$ \\
\hline $47-51$ & 2013 May 24 & $1,3,5,7,9$ & $\mathrm{Ne}$ & 3 & $2200-3900$ & 250,000 & 60 & 90 \\
\hline $52-56$ & 2013 May 21 & $1,3,5,7,9$ & $\mathrm{Ne}$ & 5 & $2200-3900$ & 250,000 & 90 & 60 \\
\hline $57-61$ & 2013 May 22 & $1,3,5,7,9$ & $\mathrm{Ne}$ & 10 & $2200-3900$ & 250,000 & 90 & 60 \\
\hline $62-66$ & 2013 May 23 & $1,3,5,7,9$ & $\mathrm{Ne}$ & 15 & $2200-3900$ & 250,000 & 60 & 90 \\
\hline $67-71$ & 2013 May 20 & $1,3,5,7,9$ & $\mathrm{Ar}$ & 3 & $2200-3900$ & 250,000 & 6 & 900 \\
\hline $77-81$ & 2013 May 16 & $1,3,5,7,9$ & $\mathrm{Ar}$ & 10 & $2200-3900$ & 250,000 & 88 & 60 \\
\hline $82-86$ & 2013 May 17 & $1,3,5,7,9$ & $\mathrm{Ar}$ & 12 & $2200-3900$ & 250,000 & 60 & 90 \\
\hline 87-91 & 2014 Jan 30 & $1,3,5,7,9$ & $\mathrm{Ne}$ & 5 & $2000-2800$ & 250,000 & 18 & 300 \\
\hline $92-96$ & 2014 Jan 31 & $1,3,5,7,9$ & $\mathrm{Ne}$ & 10 & $2000-2800$ & 250,000 & 36 & 150 \\
\hline $97-101$ & 2014 Feb 3 & $1,3,5,7,9$ & $\mathrm{Ne}$ & 15 & $2000-2800$ & 250,000 & 72 & 75 \\
\hline $102-106$ & 2014 Feb 5 & $1,3,5,7,9$ & $\mathrm{Ar}$ & 15 & $2000-2800$ & 250,000 & 45 & 120 \\
\hline $127-131$ & 2014 May 13 & $1,3,5,7,9$ & $\mathrm{Ar}$ & 10 & $2100-3200$ & 250,000 & 40 & 180 \\
\hline $137-141$ & 2014 May 14 & $1,3,5,7,9$ & $\mathrm{Ne}$ & 15 & $2100-3200$ & 250,000 & 120 & 45 \\
\hline
\end{tabular}

Notes. ${ }^{a}$ At least 3 CCD frames are needed to capture a complete echelle grating order in the UV. In the above data 5 CCD frames are used to provide redundancy and a check for lamp drift.

Ar lines. In addition, spectra are needed with the lamp operating over a range of current. Overlapping visible-UV and IR spectra of the lamps operating at high current are needed for high $\mathrm{S} / \mathrm{N}$ measurements on very weak branches to all known lower levels. Conversely, one also needs visible-UV spectra of the lamps operating at low currents in which the dominant branches are optically thin. Optical depth errors can still be present even for the lowest current FTS spectra used in this study, and these concerns are addressed using the echelle spectrometer as described in Section 3.

A relative radiometric calibration of the FTS spectra is essential for the measurement of accurate emission branching fractions. As in our past branching fraction studies we make use of the ArI and Ar II line calibration technique. Sets of well-known branching ratios for Ar I and Ar II lines have been established for this purpose in the $4300-35,000 \mathrm{~cm}^{-1}$ range by Adams \& Whaling (1981), Danzmann \& Kock (1982), Hashiguchi \& Hasikuni (1985), and Whaling et al. (1993). Intensities for these lines are measured and compared to known branching ratios in order to construct a relative radiometric calibration. This technique is internal to the HCD lamp and captures the wavelength-dependent response of the detectors, spectrometer optics, lamp windows, and any reflections which contribute to the measured signal. As described in the next Section, the use of an echelle spectrometer allows us to extend the branching fraction measurements beyond $35000 \mathrm{~cm}^{-1}$.

\section{ECHELLE SPECTROMETER DATA}

As mentioned in the previous section, one motivation for the construction of an echelle spectrometer at the University of Wisconsin is the closure of the NSO $1.0 \mathrm{~m}$ FTS. A further motivation is the need to reduce optical depth errors as a source of systematic uncertainty in branching fraction measurements, especially on weak lines. FTS instruments suffer from inherent multiplex noise, in which quantum statistical (Poisson) noise from all spectral features, particularly strong visible and near-IR branches, is smoothly redistributed throughout the entire spectrum. This can be a hindrance when measuring the weak UV transitions that are typically the most important for accurate $\mathrm{Fe}$-group abundance determinations. Often, as the lamp current is reduced, the weak lines become comparable to the multiplex noise before the dominant branch(es) from the common upper level are optically thin. The dispersive echelle spectrometer is free from multiplex noise and provides adequate $\mathrm{S} / \mathrm{N}$ on these astrophysically important weak lines even at very low lamp current, reducing the possibility of optical depth errors.

The new spectrometer incorporates a $3.0 \mathrm{~m}$ focal length grating spectrograph with a large $(128 \times 254 \mathrm{~mm}$ ruled area $)$, coarse (23.2 grooves $\mathrm{mm}^{-1}$ ) echelle grating with a 63.5 blaze angle. Attached to the spectrograph is a custom $0.5 \mathrm{~m}$ focal length orthogonal order separator, which generates and images a 2D spectrum onto a large UV-sensitive CCD array while also serving to compensate aberrations in the spectrograph. The echelle spectrometer has a resolving power of 250,000, broad wavelength coverage, and superb UV sensitivity. While the echelle spectrometer has lower resolution and wavenumber precision compared to a FTS, it has the main advantage of being free from multiplex noise. This allows us to record spectra of commercial sealed hollow cathode discharge (HCD) lamps operating at very low currents, in order to eliminate optical depth errors, while still being able to detect weak lines with adequate $\mathrm{S} / \mathrm{N}$. Two HCD lamps are used, one each with Ar and Ne buffer gases, to check for and eliminate possible blends. A more detailed description of the echelle spectrometer, including a thorough aberration analysis, is presented by Wood \& Lawler (2012).

In addition to the 13 FTS spectra listed in Table 1, the 75 CCD frames of echelle spectrometer spectra listed in Table 2 are also part of this V II transition probability study. These spectra are radiometrically calibrated using the UV continuum from a NIST-traceable $\mathrm{D}_{2}$ standard lamp. This lamp is periodically checked against a NIST-calibrated Ar mini-arc to ensure an accurate UV calibration. The use of standard lamps to calibrate a FTS is often difficult due to ghosts, and we instead rely upon the calibration method described in Section 2, but it is our preferred method for calibrating the echelle spectrometer. The use of a $\mathrm{D}_{2}$ standard lamp also enables branching fraction measurements to wavelengths shorter than that allowed using the ArI and Ar II branching ratio technique since the standard lamp is calibrated to $2000 \AA$. 
Table 3

Experimental Atomic Transition Probabilities for 203 Lines of V II from Upper Odd Parity Levels Organized by Increasing Wavelength in Air

\begin{tabular}{|c|c|c|c|c|c|c|}
\hline \multirow{2}{*}{$\begin{array}{l}\text { Wavelength } \\
\text { in Air }{ }^{\mathrm{a}} \\
(\AA)\end{array}$} & \multicolumn{2}{|c|}{ Upper Level $^{\mathrm{b}}$} & \multicolumn{2}{|c|}{ Lower Level $^{\mathrm{b}}$} & \multirow{2}{*}{$\begin{array}{c}\text { Transition } \\
\text { Probability } \\
\left(10^{6} \mathrm{~s}^{-1}\right)\end{array}$} & \multirow[t]{2}{*}{$\log _{10}(g f)$} \\
\hline & $\begin{array}{l}\text { Energy } \\
\left(\mathrm{cm}^{-1}\right)\end{array}$ & $J$ & $\begin{array}{l}\text { Energy } \\
\left(\mathrm{cm}^{-1}\right)\end{array}$ & $J$ & & \\
\hline 2123.3231 & 47420.230 & 4 & 339.125 & 4 & $5.8 \pm 1.0$ & -1.45 \\
\hline 2126.9227 & 47108.079 & 1 & 106.643 & 1 & $5.8 \pm 0.9$ & -1.93 \\
\hline 2129.4687 & 47051.889 & 3 & 106.643 & 1 & $5.9 \pm 1.1$ & -1.55 \\
\hline 2131.8351 & 47101.932 & 2 & 208.790 & 3 & $9.1 \pm 1.3$ & -1.51 \\
\hline 2134.0805 & 46879.911 & 2 & 36.102 & 1 & $13.3 \pm 2.1$ & -1.34 \\
\hline
\end{tabular}

Notes.

a Wavelength values computed from energy levels using the standard index of air from Peck \& Reeder (1972).

${ }^{\mathrm{b}}$ Energy levels, parities, and $J$ values are from Thorne et al. (2013).

(This table is available in its entirety in a machine-readable form in the online journal. A portion is shown here for guidance regarding its form and content.)

\section{V II BRANCHING FRACTIONS}

All possible transition wavenumbers between known energy levels of V II from Thorne et al. (2013) that satisfy both the parity change and $|\Delta J| \leqslant 1$ selection rules are computed and used during this branching fraction analysis. Transitions which violate these two selection rules are suppressed by a factor of $\sim 10^{6}$ and are unimportant for stellar abundance studies. These two selection rules are obeyed throughout the periodic table whereas many important Fe-group transitions violate the $\Delta S=$ 0 and $|\Delta L| \leqslant 1$ selection rules of $L S$ coupling. We can make measurements for branching fractions as weak as 0.0001 , and therefore systematic errors from missing branches are negligible in this study.

Branching fraction measurements are completed for all 31 odd-parity upper levels with LIF lifetime measurements by Den Hartog et al. (2014). In addition, branching fractions are measured for the odd-parity upper level at $39403.787 \mathrm{~cm}^{-1}$, which has previously reported lifetime measurements from Biémont et al. (1989) and Xu et al. (2006). As in our previous work, thousands of possible spectral line observations are analyzed in both FTS and echelle spectra to calculate the branching fractions. Integration limits and nonzero baselines are set "interactively" during data analysis. Nonzero baselines are necessary for the echelle spectra, which are not background corrected, and are occasionally needed for the FTS spectra when a line falls on the wing of a dominant feature. A simple numerical integration technique is used to determine un-calibrated V II line intensities. This method is preferred since the majority of V II lines have unresolved hyperfine structure that leads to variations in the observed line width. For consistency, this method is also applied to lines with partially resolved hyperfine structure. This same integration technique is also used on selected Ar I and Ar II lines to establish a relative radiometric calibration for each of the FTS spectra.

Branching fraction uncertainties depend on the $\mathrm{S} / \mathrm{N}$ of the data, the line strengths, and the wavelength separation of lines from a common upper level. Branching fraction uncertainty always migrates to the weakest lines because branching fractions sum to 1.0 by definition. Uncertainties on weak lines near the dominant branch(es) from the common upper level tend to be limited by $\mathrm{S} / \mathrm{N}$. For lines that are widely separated in wavelength from the dominant branch(es), systematic errors in the radiometric calibration tend to be the dominant source of uncertainty. The systematic uncertainty in the calibration is estimated using the product of $0.001 \% / \mathrm{cm}^{-1}$ and the wavenumber difference between the line of interest and the dominant branch from the common upper level, as presented and tested by Wickliffe et al. (2000). The calibration uncertainty is combined with the standard deviation of measurements from multiple spectra to determine the total branching fraction uncertainty. The final uncertainty, especially for lines widely separated from the dominant branch(es) from a common upper level, is primarily systematic and it is therefore impractical to state whether it represents $1 \sigma$ or $2 \sigma$ error bars. The combination of data from both the FTS and echelle spectrometer, which make use of independent radiometric calibration methods, is important in assessing and controlling systematic uncertainties.

\section{V II TRANSITION PROBABILITIES AND COMPARISON TO EARLIER MEASUREMENTS}

Absolute transition probability measurements are given for 203 lines of V II in Table 3. Branching fraction measurements from a combination of FTS and echelle data are normalized with published LIF radiative lifetimes (Den Hartog et al. 2014) to determine the transition probabilities. Air wavelengths in the table are computed from V II energy levels (Thorne et al. 2013) using the standard index of air (Peck \& Reeder 1972).

Often lines must be omitted if they are too weak to have reliable $\mathrm{S} / \mathrm{N}$, have uncertain classifications, or are too seriously blended to be separated. The effect of these problem lines can be seen by summing all transition probabilities for a given upper level in Table 3 and comparing the sum to the inverse upper level lifetime (Den Hartog et al. 2014). The sum is typically $>90 \%$ of the inverse level lifetime. While these problem lines have large fractional uncertainty in their branching fractions, this does not have a significant effect on the uncertainties of the lines kept in Table 3 . The transition probability uncertainties quoted in Table 3 are found by combining branching fraction uncertainties and radiative lifetime uncertainties in quadrature.

Figures 1-3 compare our new V II transition probability data to the NIST Atomic Spectra Database as of 2014 June 11 (Kramida et al. 2013; see footnote 5). The figures are distinguished by the accuracy ratings assigned to each value in the database. Figure 1 is a comparison of $62 \log (g f)$ values in common to this work and to NIST database values with a "B" $(\leqslant 10 \%)$ accuracy rating, plotted as a function of wavelength in the upper panel (a) and the $\log (g f)$ value reported in this study in the lower panel (b). Individual error bars on the $\log (g f)$ differences represent the uncertainties on measurements from this work. The central solid line represents perfect agreement 

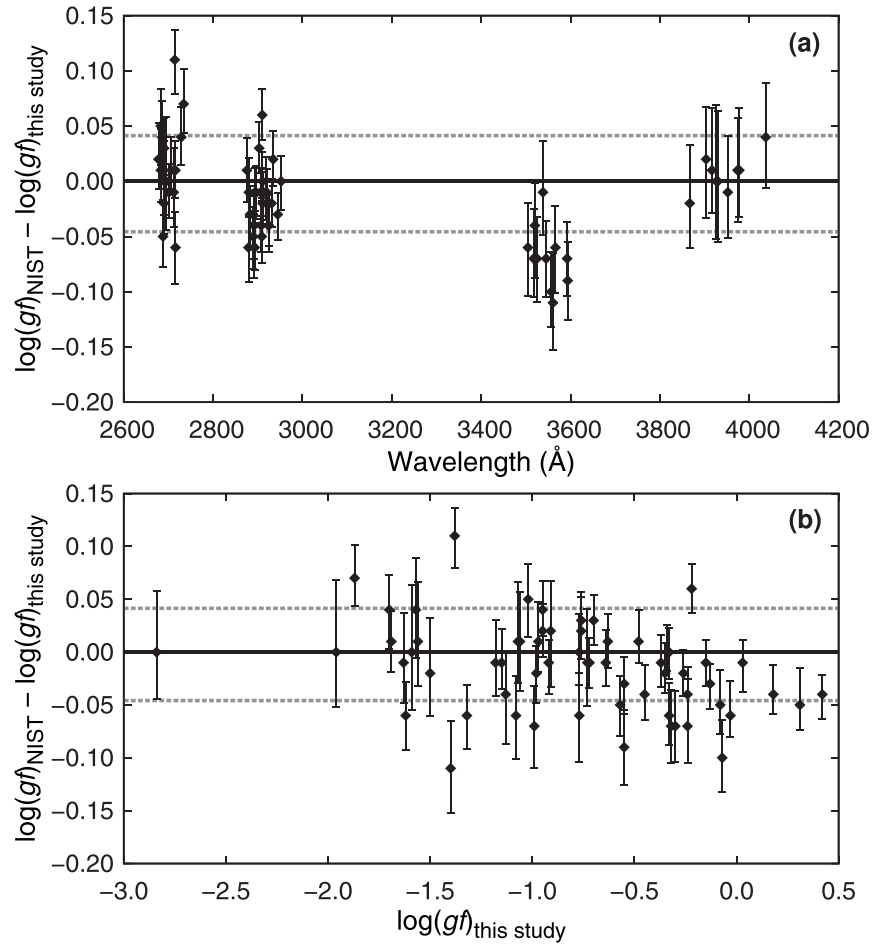

Figure 1. Comparison of $62 \log (g f)$ values with accuracy rank "B" $(\leqslant 10 \%)$ from the NIST ASD by Kramida et al. (2013) to results of this work. The $\log (g f)$ differences are plotted as a function of wavelength in the upper panel (a) and as a function of the $\log (g f)$ value reported in this study in the lower panel (b). The solid central line represents perfect agreement, the gray dashed lines indicate $\pm 10 \%$ differences in $f$ values, and the error bars are from this work only.
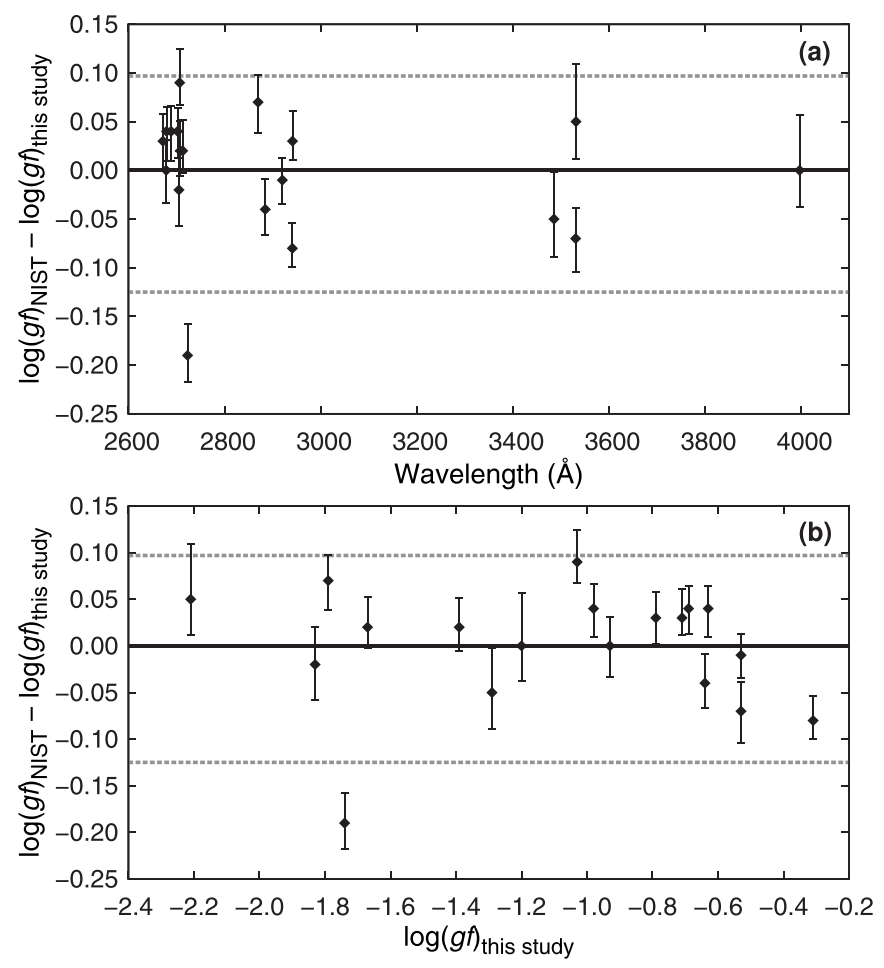

Figure 2. Comparison of $19 \log (g f)$ values with accuracy rank "C" $(\leqslant 18 \%)$ or "C+" ( $\leqslant 25 \%)$ from the NIST ASD by Kramida et al. (2013) to results of this work. The $\log (g f)$ differences are plotted as a function of wavelength in the upper panel (a) and as a function of the $\log (g f)$ value reported in this study in the lower panel (b). The solid central line and error bars have the same meaning as in Figure 1, while the gray dotted lines represent $\pm 25 \%$ differences in $f$ values.
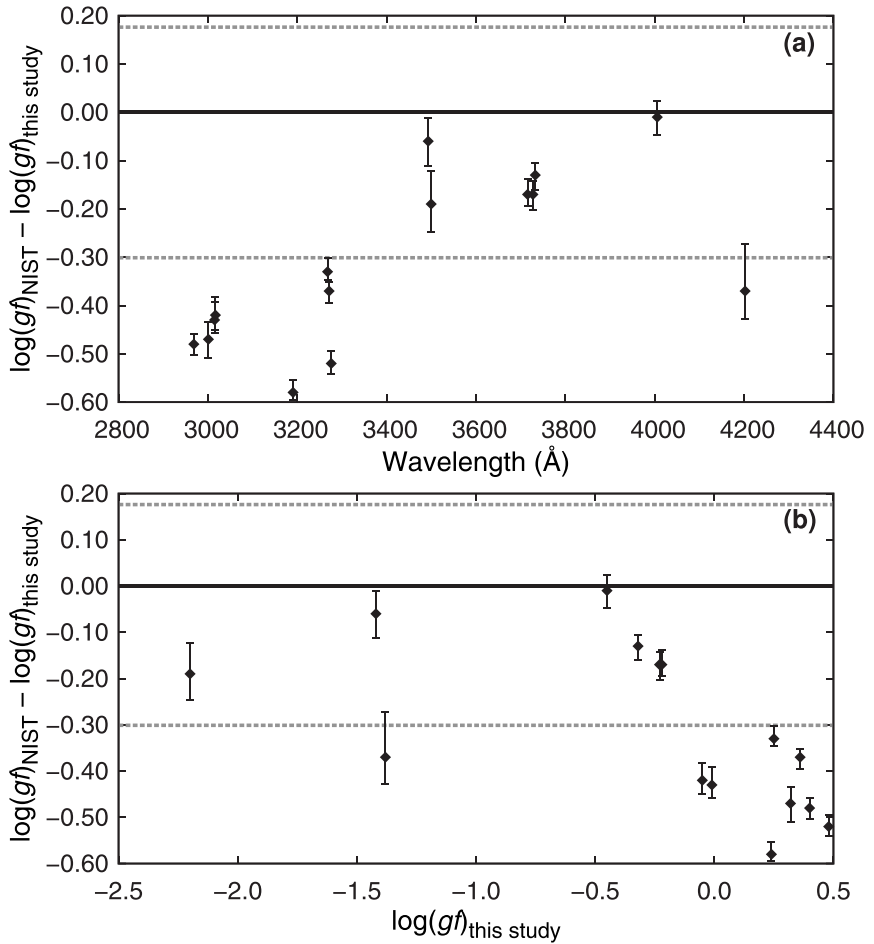

Figure 3. Comparison of $15 \log (g f)$ values with accuracy rank "D" $(\leqslant 50 \%)$ from the NIST ASD by Kramida et al. (2013) to results of this work. The $\log (g f)$ differences are plotted as a function of wavelength in the upper panel (a) and as a function of the $\log (g f)$ value reported in this study in the lower panel (b). The solid central line and error bars have the same meaning as in Figure 1, while the gray dotted lines represent $\pm 50 \%$ differences in $f$ values.

at a logarithmic difference of zero, while the grey dotted lines represent $\pm 10 \%$ differences in $f$ values. Figure 2 is a comparison of 19 lines in common between this work and the NIST database having accuracy grades of "C+" $(\leqslant 18 \%)$ or " $\mathrm{C}$ " $(\leqslant 25 \%)$, again plotted as a function of wavelength in the upper panel (a) and the $\log (g f)$ value reported in this study in the lower panel (b). The error bars and central solid line have the same meaning as in Figure 1, while the gray dotted lines represent $\pm 25 \%$ differences in $f$ values. Karamatskos et al. (1986) are cited as the source of the "B", "C+", and "C" rated data in the NIST database. Similarly to this work, those authors used a combination of LIF lifetimes and emission branching fractions to determine their transition probabilities. Aside from a few outliers, the majority of lines agree within combined uncertainties. The slight dip in Figure 1(a) for lines near $3550 \AA$ was observed previously by Biémont et al. (1989), who suggested it results from an incorrect calibration of the FTS spectra used by Karamatskos et al.

A comparison of $15 \log (g f)$ values in common to this work and the NIST database with accuracy grades of "D" $(\leqslant 50 \%)$ is plotted as a function of wavelength in the upper panel (a) of Figure 3 and the $\log (g f)$ value measured in this work in the lower panel (b). The error bars and central solid line have the same meaning as in Figure 1, whereas the gray dotted lines represent $\pm 50 \%$ differences in $f$ values. Both the work of Wujec \& Musielok (1986) and the earlier work of Roberts et al. (1973) are cited as the sources of the " $D$ " rated values in the NIST database. Roberts et al. determined their $\log (g f)$ values from a combination of lifetimes measured using the beam-foil technique with branching fractions measured from an arc discharge. Wujec \& Musielok (1986) also performed branching fraction measurements using an arc discharge, with 

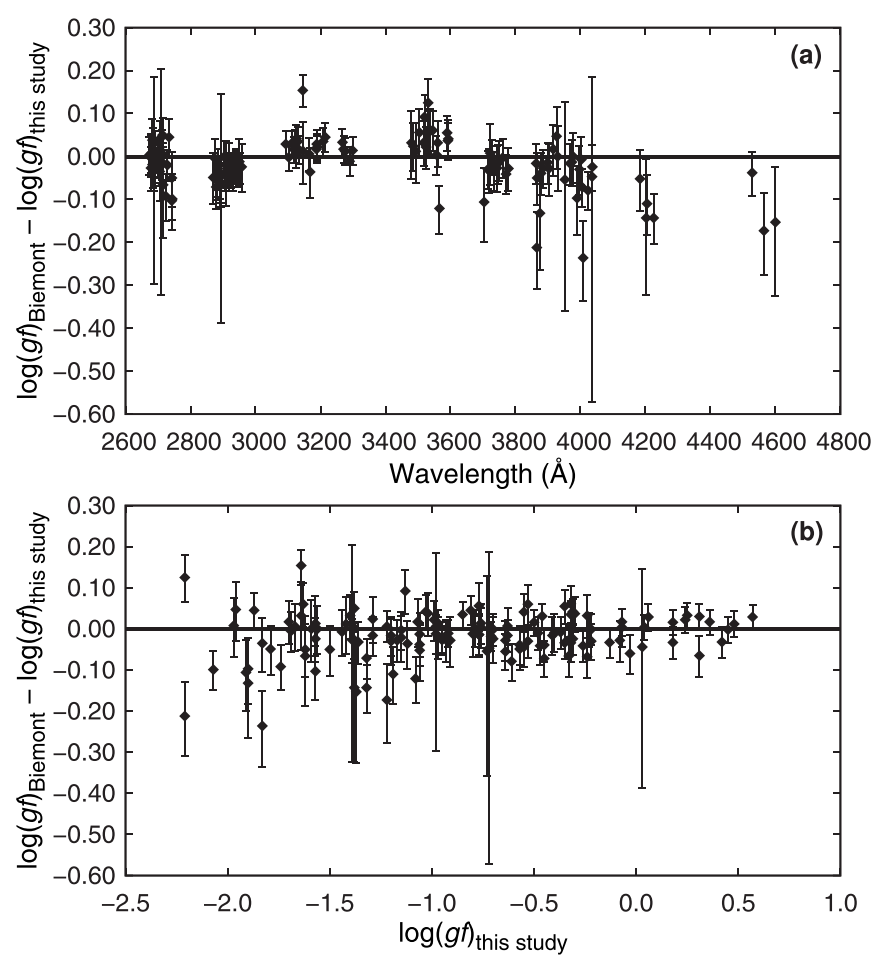

Figure 4. Comparison of $137 \log (g f)$ values in common between this work and the work of Biémont et al. (1989) plotted as a function of wavelength in the upper panel (a) and as a function of the $\log (g f)$ value reported in this work in the lower panel (b). The solid line represents perfect agreement, and the error bars represent the uncertainties reported by Biémont et al. (1989) and the uncertainties reported in this work combined in quadrature.

some $\log (g f)$ values determined using the Roberts et al. lifetimes, while other values were determined using a Boltzmann analysis to set relative $\log (g f)$ values on an absolute scale. The agreement between this work and previous measurements is not as good as in Figures 1 and 2 . The overall trend of higher $\log (g f)$ values in this work can be explained by our use of the new LIF lifetime measurements of Den Hartog et al. (2014), which in many cases are lower than the lifetimes of Roberts et al. Biémont et al. (1989) noted that the beam-foil lifetimes of Roberts et al. are long by as much as a factor of two, which they attribute to the possibility of cascading transitions in the beam-foil excitation. In addition, the tendency for stronger lines to be enhanced compared to weaker lines, as seen in Figure 3(b), is evidence for optical depth errors in the earlier measurements. This current work makes use of a new echelle spectrometer which was specifically developed to address optical depth concerns in transition probability measurements.

Figure 4 is a comparison of $\log (g f)$ values for 137 lines in common to this work and the work of Biémont et al. (1989), plotted as a function of wavelength in the upper panel (a) and the $\log (g f)$ value measured in this work in the lower panel (b). The solid line represents perfect agreement at a logarithmic difference of 0 , while the error bars represent the uncertainty reported by Biémont et al. (1989) and the uncertainty reported herein combined in quadrature. Similarly to this work, Biémont et al. used a combination of LIF radiative lifetimes and emission branching fractions from FTS spectra to determine the majority of their transition probabilities. Transition probabilities for two additional levels were measured by interpolating upper level populations in an inductively coupled plasma source. While six of the LIF lifetimes were new measurements (including the lifetime value utilized in this study for the $39403.787 \mathrm{~cm}^{-1}$ upper level), Biémont et al. also utilized earlier lifetime measurements from Karamatskos et al. (1986). As in this work, spectra were recording using the NSO $1.0 \mathrm{~m}$ FTS, and as such there is some overlap in the FTS spectra used by Biémont et al. and in this work (e.g., Index \#6 in Table 1). Over $70 \%$ of the lines plotted in Figure 4 agree within combined uncertainties. However, it is likely that the agreement is actually better than this. A significant number of the transition probabilities in Table 2 of the Biémont et al. work have quoted uncertainties less than the uncertainties on the corresponding level lifetimes used to derive the transition probability values. As stated in Biémont et al., the transition probability uncertainties contain contributions from the lifetime uncertainties, $\mathrm{S} / \mathrm{N}$ effects, and uncertainty in the FTS calibration. For these transition probability values, we substitute the corresponding lifetime uncertainty in place of the quoted transition probability uncertainty to determine the error bars, since the lifetime uncertainty represents a lower limit on the transition probability uncertainty. It is likely the true uncertainties for these lines are larger than this, which would increase the size of the combined error bars and bring more lines into agreement.

\section{MEASUREMENTS OF V II HYPERFINE STRUCUTRE CONSTANTS}

Vanadium is essentially monoisotopic, with ${ }^{51} \mathrm{~V}$ being the only naturally occurring stable ${ }^{8}$ isotope, and therefore isotope shifts are unimportant for this study. However, since ${ }^{51} \mathrm{~V}$ has a nonzero nuclear spin $(I=7 / 2)$, hyperfine structure (HFS) leads to a broadening of many V II transitions observed in this study. Several V II levels have previously reported experimental HFS $A$ constants. Arvidsson (2003) measured 26 A constants using least squares fitting of HFS patterns in two FTS spectra, one recorded using the NSO $1.0 \mathrm{~m}$ FTS (likely \#7 in our Table 1) and an additional FTS spectrum, recorded at Lund University, to capture the deep UV. More recently, Armstrong et al. (2011) published a set of 55 high accuracy HFS $A$ constants from LIF measurements made using a single-frequency laser on a beam of $\mathrm{V}$ atoms.

Similarly to Arvidsson (2003), in this work we use least squares fitting of V II line profiles in order to determine new HFS $A$ constants. We make use of the Casimir formula, as presented in the text by Woodgate (1980),

$$
\Delta E=\frac{A}{2} K+\frac{B}{8} \frac{3 K(K+1)-4 I(I+1) J(J+1)}{I(2 I-1) J(2 J-1)},
$$

where $\Delta E$ is the wavenumber shift of an HFS sublevel $(F, J)$ from the center of gravity of the fine-structure level $(J)$,

$$
K=F(F+1)-J(J+1)-I(I+1),
$$

$F$ is the total atomic angular momentum, $J$ is the total electronic angular momentum, and $I$ is the nuclear spin. Unfortunately the FTS spectra we utilize do not have adequate resolution or $\mathrm{S} / \mathrm{N}$, or both, in order to determine any HFS $B$ constants,

\footnotetext{
${ }^{8}{ }^{50} \mathrm{~V}$ is nearly stable, with a half-life of $\sim 10^{17} \mathrm{yr}$. However, its solar-system abundance is only $0.25 \%$, entirely negligible for this study.
} 
Table 4

New V II Magnetic Dipole Hyperfine Structure Constants from FTS Spectra

\begin{tabular}{|c|c|c|c|c|c|c|}
\hline \multirow[t]{2}{*}{ Configuration $^{\mathrm{a}}$} & \multirow[t]{2}{*}{ Term $^{\mathrm{a}}$} & \multirow[t]{2}{*}{$J^{\mathrm{a}}$} & \multirow[t]{2}{*}{ Parity $^{\mathrm{a}}$} & \multirow{2}{*}{$\begin{array}{c}\text { Level Energy }{ }^{\mathrm{a}} \\
\qquad\left(\mathrm{cm}^{-1}\right)\end{array}$} & \multicolumn{2}{|c|}{$\begin{array}{l}\text { Hyperfine Structure Constant } A \\
\qquad\left(10^{-3} \mathrm{~cm}^{-1}\right)\end{array}$} \\
\hline & & & & & This Work & Arvidsson $^{\mathrm{b}}$ \\
\hline $3 d^{3}\left({ }^{4} \mathrm{~F}\right) 4 s$ & $a^{5} F$ & 1 & ev & 2605.040 & $-13.7 \pm 1.0$ & $17.0 \pm 5.7$ \\
\hline $3 d^{3}\left({ }^{4} \mathrm{~F}\right) 4 s$ & $a^{5} F$ & 2 & ev & 2687.208 & $13.5 \pm 3.0$ & $11.1 \pm 1.9$ \\
\hline $3 d^{3}\left({ }^{4} \mathrm{~F}\right) 4 s$ & $a^{5} \mathrm{~F}$ & 3 & ev & 2808.959 & $18.4 \pm 1.0$ & $19.8 \pm 2.0$ \\
\hline $3 d^{3}\left({ }^{4} \mathrm{~F}\right) 4 s$ & $a^{5} F$ & 4 & ev & 2968.389 & $20.5 \pm 1.0$ & $27.6 \pm 1.9$ \\
\hline $3 d^{3}\left({ }^{4} \mathrm{~F}\right) 4 s$ & $a^{5} F$ & 5 & ev & 3162.966 & $21.8 \pm 1.0$ & $24.4 \pm 1.5$ \\
\hline $3 d^{3}\left({ }^{4} \mathrm{~F}\right) 4 s$ & $a^{3} \mathrm{~F}$ & 2 & ev & 8640.362 & $30.5 \pm 1.0$ & $31.3 \pm 0.2$ \\
\hline $3 d^{3}\left({ }^{4} \mathrm{~F}\right) 4 s$ & $a^{3} F$ & 3 & ev & 8842.050 & $6.0 \pm 1.0$ & \\
\hline $3 d^{3}\left({ }^{4} \mathrm{~F}\right) 4 s$ & $a^{3} F$ & 4 & ev & 9097.889 & $-2.9 \pm 1.0$ & \\
\hline $3 d^{4}$ & $a^{3} P$ & 1 & ev & 11514.784 & $0.0 \pm 2.0$ & \\
\hline $3 d^{4}$ & $\mathrm{a}^{3} \mathrm{P}$ & 2 & ev & 11908.261 & $0.0 \pm 2.0$ & \\
\hline $3 d^{4}$ & $a^{3} G$ & 3 & ev & 14461.748 & $5.0 \pm 1.0$ & \\
\hline $3 d^{3}\left({ }^{4} \mathrm{~F}\right) 4 p$ & $z^{5} G$ & 2 & od & 34592.843 & $26.0 \pm 1.5$ & \\
\hline $3 d^{3}\left({ }^{4} \mathrm{~F}\right) 4 p$ & $z^{5} G$ & 3 & od & 34745.828 & $14.4 \pm 1.5$ & \\
\hline $3 d^{3}\left({ }^{4} \mathrm{~F}\right) 4 p$ & $z^{5} G$ & 4 & od & 34946.637 & $9.0 \pm 1.5$ & \\
\hline $3 d^{3}\left({ }^{4} \mathrm{~F}\right) 4 p$ & $z^{5} G$ & 5 & od & 35193.182 & $6.7 \pm 1.5$ & \\
\hline $3 d^{3}\left({ }^{4} \mathrm{~F}\right) 4 p$ & $z^{5} G$ & 6 & od & 35483.606 & $5.2 \pm 2.0$ & \\
\hline $3 d^{3}\left({ }^{4} \mathrm{~F}\right) 4 p$ & $z^{5} \mathrm{~F}$ & 3 & od & 36919.266 & $4.9 \pm 1.5$ & \\
\hline $3 d^{3}\left({ }^{4} \mathrm{~F}\right) 4 p$ & $z^{5} F$ & 4 & od & 37150.615 & $4.9 \pm 1.5$ & \\
\hline $3 d^{3}\left({ }^{4} \mathrm{~F}\right) 4 p$ & $z^{5} D$ & 3 & od & 37520.665 & $2.5 \pm 1.5$ & \\
\hline $3 d^{3}\left({ }^{4} \mathrm{~F}\right) 4 p$ & $\mathrm{z}^{5} \mathrm{P}$ & 2 & od & 46879.911 & $-4.2 \pm 1.5$ & \\
\hline $3 d^{3}\left({ }^{4} \mathrm{~F}\right) 4 p$ & $\mathrm{z}^{5} \mathrm{P}$ & 3 & od & 47051.889 & $1.1 \pm 1.5$ & \\
\hline
\end{tabular}

Notes.

${ }^{a}$ Level energies and classifications are from Thorne et al. (2013).

b Arvidsson (2003) master's thesis at Lund University, Lund, Sweden.

and therefore we neglect the electric quadrupole interaction term in determining the energy shifts. For this same reason, rather than taking a broad approach and measuring as many new HFS $A$ constants as possible, we instead target transitions which are used in either the abundance analyses of the Sun (Section 7) or HD 84937 (Section 8). We choose to focus on lines that are broadened and/or show HFS in our FTS spectra and cause a nonnegligible amount of broadening in the solar and stellar spectra, as we are able to obtain the most reliable results for these lines. For these transitions, we start by using the LIF measurements of Armstrong et al. (2011) to fix either the upper or lower level HFS $A$ constant, and then nonlinear least-squares fit the observed HFS pattern in order to determine the HFS $A$ constant for the other level. By fixing these newly measured HFS $A$ constants, we can then proceed to fit HFS patterns for transitions which have neither an upper or lower LIF HFS constant measurement. In addition to the HFS $A$ constants of the upper or lower level, the fitting parameters include the center of gravity wavenumber, the total intensity of the line, and one line-profile parameter which represents the convolution of the instrumental sinc function with a variable-temperature Doppler-broadened Gaussian function.

Table 4 lists 21 new magnetic dipole HFS $A$ constants measured in this study. These values are determined from a $\mathrm{S} / \mathrm{N}$ weighted mean of HFS pattern fits in four FTS spectra (\#1, 2, 3, and 13 in Table 1), while error bars represent the standard deviation of the measurements. In general there is good agreement with the earlier work of Arvidsson (2003), with a few exceptions. This work benefits from newly measured level energies and classifications (Thorne et al. 2013), which help identify possible blends, as well as being tied to new and accurate LIF HFS constants from Armstrong et al. (2011), so we are confident in our reported values. Please note that while the HFS $A$ constants from Armstrong et al. are not listed in
Table 4, they did serve as reference values in our study due to the high spectral resolution and $\mathrm{S} / \mathrm{N}$ of their single frequency laser measurements. For the level at $2605.040 \mathrm{~cm}^{-1}$, the agreement is significantly worse, with our new result having almost equal magnitude but opposite sign to that reported by Arvidsson (2003). However, the value presented here is reinforced by new theoretical relativistic configuration interaction calculations from Beck \& Abdalmoneam (2014). Using the LIF HFS measurements of Armstrong et al. (2011) in combination with the new HFS A constants from Table 4, HFS component patterns for selected lines used in the solar and/or HD 84973 V II abundance determinations are listed in Table 5. Several patterns listed in Table 5 connect to the ground term, even though these levels have no previously reported HFS $A$ constants and are not included in Table 4. While we are unable to reliably measure the HFS constants for these levels, we conclude from the FTS spectra that they are small, and for the purposes of Table 5 the HFS $A$ constants for levels in the ground term have been set to zero. Individual energy shifts are calculated using the Casimir formula quoted above and the component strengths are normalized to sum to unity. Given the relatively large error bars on some of the newly measured HFS $A$ constants listed in Table 4, we choose not to attempt HFS pattern determinations on lines which are not needed in the abundance analyses presented in Sections 7 and 8. Therefore we caution that Table 5 is not meant to be an exhaustive list of lines with measurable HFS in V II, and there may be other lines with detectable HFS broadening.

\section{THE VANADIUM ABUNDANCE IN THE SOLAR PHOTOSPHERE}

We apply our new V II transition probability and HFS data to produce a new $\mathrm{V}$ abundance for the solar photosphere. 
Table 5

Hyperfine Structure Line Component Patterns for 67 Lines of V II

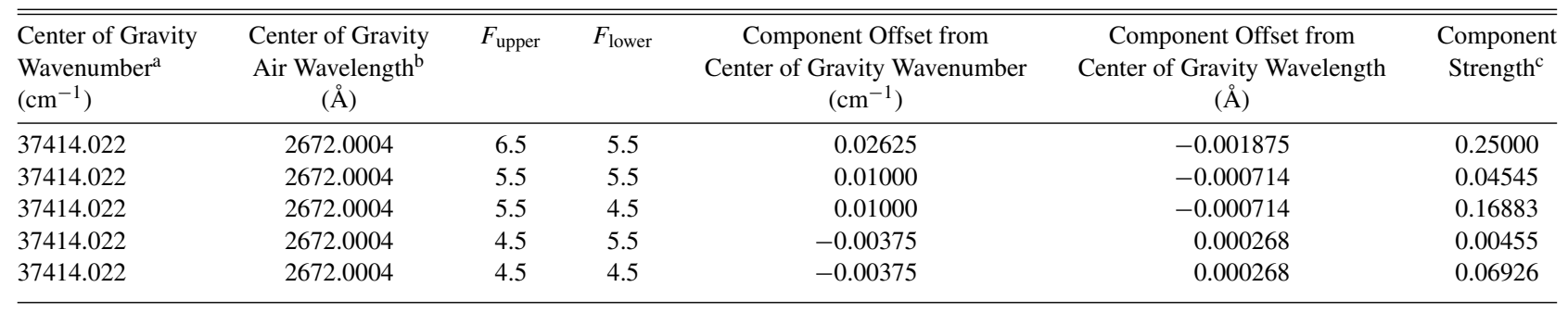

Notes.

a Energy levels are from Thorne et al. (2013).

b Wavelength values computed from energy levels using the standard index of air from Peck \& Reeder (1972).

${ }^{c}$ Component strengths are normalized to sum to unity.

(This table is available in its entirety in a machine-readable form in the online journal. A portion is shown here for guidance regarding its form and content.)

We follow the techniques described in our previous studies of Fe-group species: Ti I (Lawler et al. 2013), Ti II (Wood et al. 2013), and Ni I (Wood et al. 2014). Since we employ synthetic spectrum analyses for each feature, substitution of full sets of HFS components for individual lines is straightforward; see Lawler et al. (2001a, 2001b) for previous examples of this procedure. In our Ni I study (Wood et al. 2014) we included the effects of isotopic wavelength shifts in our solar abundance determinations, but with only one naturally-occurring stable isotope $\left({ }^{51} \mathrm{~V}\right)$, isotopic shifts are irrelevant here. However, whereas HFS was unimportant in our Ni I study, it must be incorporated into our abundance analysis here.

To begin, as in our previous papers, we estimate approximate V II absorption transition strengths with the simple formula,

$$
\mathrm{STR} \equiv \log (g f)-\theta \chi
$$

with the $\log (g f)$ values given in Table 3, excitation energies $\chi(\mathrm{eV})$, and inverse temperature $\theta=5040 / T$ (we assume $\theta=$ 1.0 for this rough calculation). The STR values are plotted as a function of wavelength in Figure 5. Red circles call attention to those lines that we use in the solar abundance computations (see below). These relative strengths apply only to V II because they include neither Saha ionization factors (which could allow comparison to other vanadium species) nor the vanadium solar abundance (which could allow comparison to other elements). They do, however, indicate the relative line strengths for V II lines. In Figure 5, the horizontal line at STR $=-4.1$ indicates the strengths of $\mathrm{V}_{\text {II }}$ lines that lie at the approximate weak-line limit of features that are useful in a solar abundance analysis. Expressing the line equivalent width $E W$ as $\log$ of the reduced width $\log (R W)=\log (E W / \lambda)$, the weak-line limit is approximately $\log (R W) \sim-6$. For Figure 5 the corresponding weak-line strength of -4.1 is determined empirically, by measuring the $E W \mathrm{~s}$ of the weakest $\mathrm{V}_{\text {II }}$ lines of Table 3. All of the lines in our study are stronger than $\log (R W) \sim-6$ in the solar center-of-disk spectrum (Delbouille et al. 1973) $)^{9}$. Thus if unblended they are potentially useful photospheric vanadium abundance indicators.

Table 3 lists nearly $110 \mathrm{~V}$ II lines with wavelengths longer than the atmospheric cutoff ( $3000 \AA$, indicated with the blue vertical line in Figure 5). However, almost all of these lines arise at wavelengths $\lambda<4100 \AA$, in the crowded near-UV spectral

\footnotetext{
9 http://bass2000.obspm.fr/solar_spect.php
}

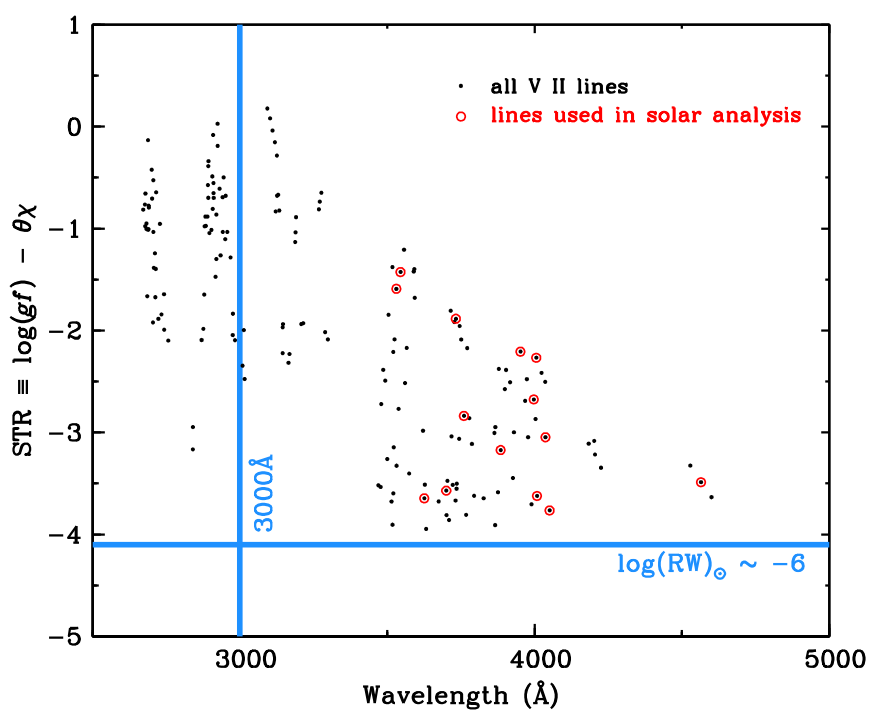

Figure 5. Relative strengths of V II lines as defined by the vertical axis label; see text for further discussion. The vertical blue line indicates the atmospheric cutoff for ground-based spectroscopy. The horizontal blue line indicates the STR values of barely detectable lines (reduced widths $\log (R W)=-6$ ). Red circles show the 15 lines used in our solar analysis.

(A color version of this figure is available in the online journal.)

region. Therefore the main impediment to their use is contamination by transitions of other atomic and molecular species. We follow the procedures of our previous papers to determine which V II lines can be employed. We inspect each line in the Delbouille et al. (1973) photospheric spectrum, and then use the Moore et al. (1966) solar line identification compendium and the Kurucz (2011) ${ }^{10}$ atomic/molecular line database to identify those V II lines that are too blended to yield trustworthy vanadium abundances. Unfortunately, this procedure eliminates the vast majority of V II lines in Table 3, and we are left with only $25 \mathrm{~V}$ II lines meriting further investigation.

We compute synthetic spectra for these surviving transitions with the current version of the LTE line analysis code MOOG $^{11}$ (Sneden 1973). Line list assembly is described in detail by Lawler et al. (2013). Briefly, we begin with the Kurucz (2011) line database, gathering atomic and $\mathrm{CN}, \mathrm{CH}, \mathrm{NH}$, and $\mathrm{OH}$ molecular lines in a typically $4 \AA$ interval centered on each

\footnotetext{
10 http://kurucz.harvard.edu/linelists.html

11 Available at http://www.as.utexas.edu/ chris/moog.html
} 
Table 6

Lines of Ionized Vanadium in the Solar Photosphere

\begin{tabular}{lcccc}
\hline \hline $\begin{array}{l}\text { Wavelength in Air } \\
(\AA)\end{array}$ & $\begin{array}{c}\text { Excitation Energy } \\
(\mathrm{eV})\end{array}$ & $\log _{10}(g f)$ & $\log _{10}(\varepsilon)$ & HFS $^{\mathrm{a}}$ \\
\hline 3530.772 & 1.070 & -0.53 & 3.95 & yes \\
3545.196 & 1.095 & -0.32 & 4.00 & yes \\
3625.611 & 2.375 & -1.22 & 3.90 & no \\
3700.125 & 2.489 & -1.11 & 3.90 & no \\
3732.748 & 1.564 & -0.32 & 3.83 & no \\
3760.221 & 1.686 & -1.15 & 3.97 & yes \\
3884.836 & 1.792 & -1.38 & 3.91 & yes \\
3866.722 & 1.427 & -1.52 & 3.95 & no \\
3951.957 & 1.475 & -0.73 & 3.95 & yes \\
3997.110 & 1.475 & -1.20 & 3.98 & no \\
4005.702 & 1.816 & -0.45 & 3.95 & yes \\
4008.162 & 1.792 & -1.83 & 3.97 & yes \\
4036.764 & 1.475 & -1.57 & 4.00 & yes \\
4051.045 & 1.803 & -1.97 & 4.03 & no \\
4564.577 & 2.266 & -1.22 & 4.03 & yes \\
\hline
\end{tabular}

Note. ${ }^{a}$ This column denotes whether or not hyperfine substructure has been included in the abundance calculation.

V II line, but modify transition probabilities and account for isotopic/hyperfine substructure as needed from recent lab studies on these species: second spectra rare earth atoms (Sneden et al. 2009, and references therein), Cr I (Sobeck et al. 2007), Ti I (Lawler et al. 2013), Ti II (Wood et al. 2014), and Ni I (Wood et al. 2014). To be consistent with our previous work beginning with Lawler et al. (2001a), we adopt the Holweger \& Müller (1974) empirical model photosphere. The line lists and solar model are used as inputs in MOOG, and the output raw synthetic spectra are then convolved with Gaussian smoothing functions to empirically match the broadening effects of the spectrograph instrument profile (a negligible effect for the Delbouille et al. 1973 solar spectral atlas) and solar macroturbulence. For V II lines and for the lines with lab transition data named above, the lab data are accepted without alteration. For other contaminants (the majority of features in most near-UV spectral windows), adjustments are made to their $\log (g f)$ values to best reproduce the observed solar photospheric spectrum.

The comparisons of observed and synthetic solar spectra result in the elimination of more V II lines, due either to unacceptably large contamination by other species or because they are simply too strong to be sensitive to abundance changes. In the end we are left with only 15 lines that are appropriate for a solar vanadium abundance determination. In Figure 6 we show observed and synthetic spectra for representative V II lines at 3530.78 and $4564.58 \AA$. This figure also shows the positions and fractional strengths of the HFS components for each line. Inclusion of HFS always serves to broaden and desaturate a transition, resulting in a derived abundance which, compared to single-line assumptions, ranges from slightly smaller (a few percent) for weak lines to factors approaching five times smaller for strong (saturated) lines.

The abundances from individual lines are listed in Table 6, in which we also include columns for line wavelengths, excitation energies, oscillator strengths, and whether HFS is included in the synthetic spectrum computations. While the majority of lines listed with a "no" in Table 6 have negligible HFS, there are some for which HFS patterns could not be determined. This may be due to a lack of resolved structure, a lack of $\mathrm{S} / \mathrm{N}$, or both in the available FTS spectra. However, the majority of lines for which HFS has a detectable effect on derived abundances have

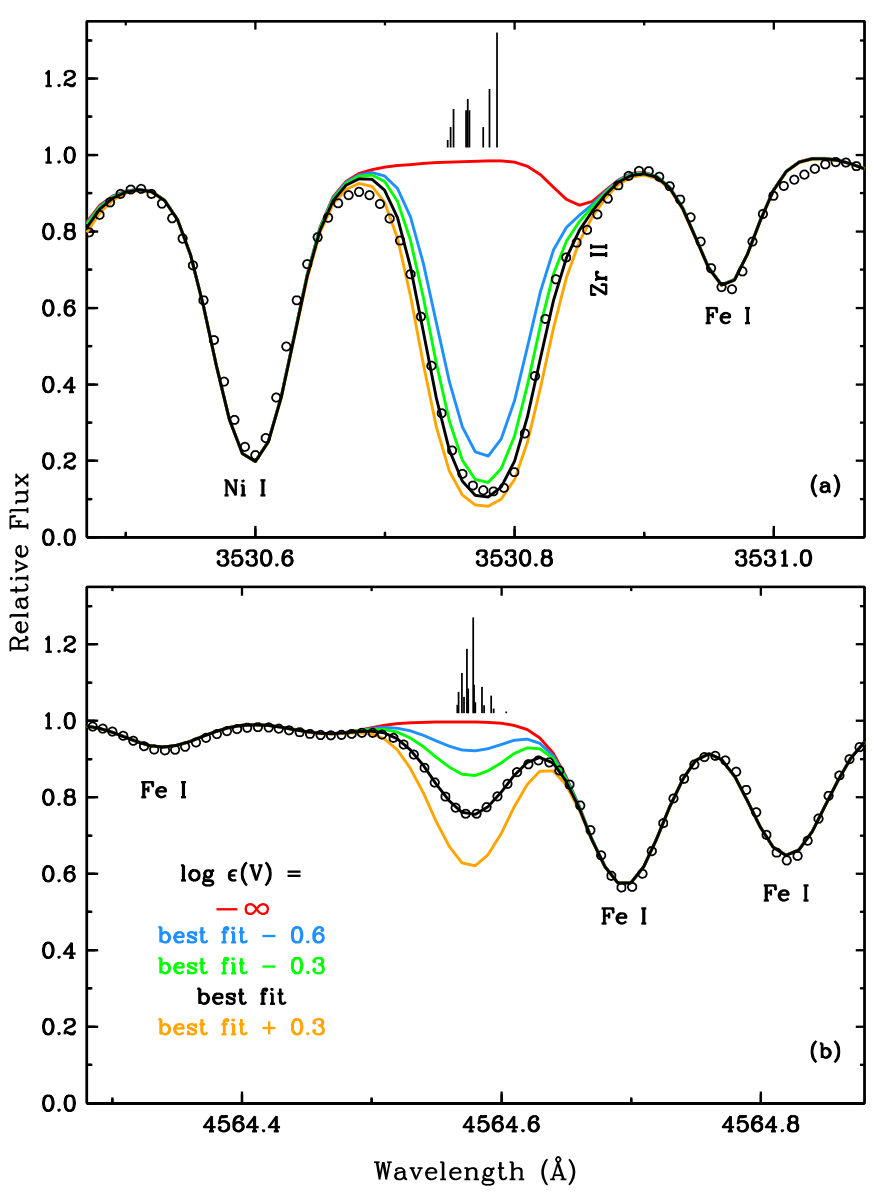

Figure 6. Synthetic and observed photospheric spectra of two V II lines. For each transition, the vertical lines indicate the wavelengths and relative strengths of the hyperfine components. These lines are placed at the component wavelengths, with vertical extents equal to their fractional contribution to the total transition probability of the total V II feature. There are 9 such components for the $3530.7 \AA$ line and 15 for the $4564.4 \AA$ line. The green open circles represent every 4 th point from the Delbouille et al. (1973) solar center-of-disk spectrum.

(A color version of this figure is available in the online journal.)

HFS patterns reported in Table 5. Inclusion of the missing HFS patterns for lines listed "no" in Table 6 would likely have a negligibly small effect on the abundance determinations. The line abundances are plotted as functions of wavelength in the top panel of Figure 7 . With this small data set we do not find any obvious trends of abundance with line wavelength, excitation energy, transition probability, or overall line strength. From these 15 lines we derive a new solar photospheric vanadium abundance: $\langle\log \varepsilon(\mathrm{V})\rangle=3.95 \pm 0.01$ with $\sigma=0.05$.

Our solar analysis unfortunately has to exclude many promis-

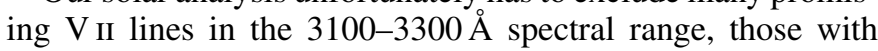
relative strengths $\mathrm{STR} \geqslant-1$ in Figure 5. These lines include ones for which we have produced syntheses but in the end must neglect, because they are so strong that their absorptions depend more on microturbulent velocity, damping, and outeratmosphere line formation physics than they do on $\mathrm{V}$ abundance. Here are four examples, giving the $\log (R W)$ values from Moore et al. (1966) in parentheses after their wavelengths: $3121.15 \AA$ (-4.38); $3126.22 \AA(-4.32) ; 3188.71 \AA(-4.45)$, and $3276.14 \AA$ $(-4.49)$. All these lines, and many others in the near-UV spectral region, are strong enough to appear on the "flat" or "damping" parts of the solar photospheric curve-of-growth. Proper accounting of HFS does not remove enough of the overall line saturation of these transitions to make them reliable abundance indicators. 

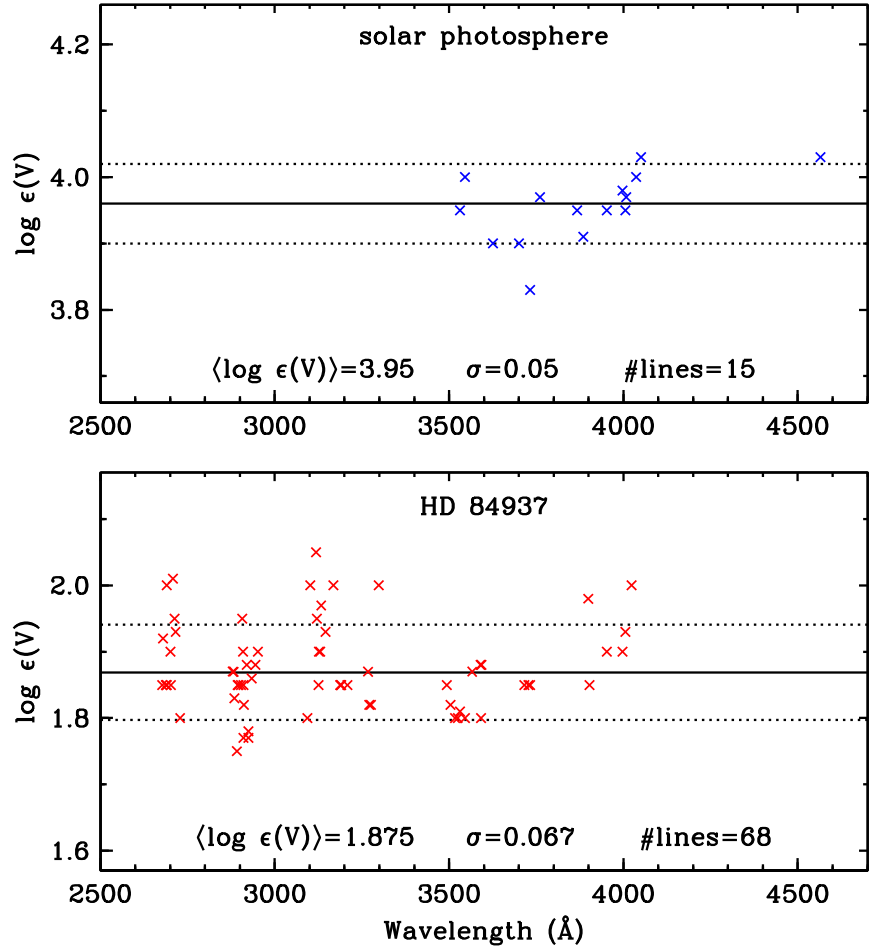

Figure 7. Vanadium abundances in the solar photosphere (top panel) and HD 84937 (bottom panel) derived from V II lines, plotted as functions of wavelength. In each panel, the solid horizontal line represents the mean abundance, and the two dotted lines are placed $\pm 1 \sigma$ from the mean. The abundance statistics are given in the panels.

(A color version of this figure is available in the online journal.)

However, our trial syntheses of these, and some other lines that are too strong for solar $\mathrm{V}$ abundance estimation, suggest that their values are compatible with the abundances derived from weaker lines. Their abundance uncertainties are simply too large to be of use here, but their great strengths will render them as prime $\mathrm{V}$ abundance indicators in very metal-poor stars.

The standard deviation of the mean solar abundance determined from the 15 lines of our study is only \pm 0.01 , and so the dominant source of total uncertainty is external, through choice of model solar atmosphere and analytical technique. Scott et al. (2014) have recently completed new analyses of the solar photospheric abundances of Fe-group elements, using EWs and a variety of solar models and line formation methodologies. They concur with previous studies that the Fe-group elements exist almost exclusively in the singly ionized species, whose absorption features can be analyzed to adequate accuracy with an assumption of LTE. For V II, Scott et al. find only five transitions amenable to $E W$ measurement, and from them derive $\langle\log \varepsilon(\mathrm{V})\rangle=4.03$ with the Holweger \& Müller (1974) solar model. But the application of other models yields essentially the same result, and the mean $\mathrm{V}$ abundance varies only in the range 3.98-4.04; see Scott et al. for extended discussion of these abundance exercises. They urge caution in interpretation of their V II result due to the few lines that they trusted for this species.

Our mean photospheric abundance of $\langle\log \varepsilon(\mathrm{V})\rangle=3.95$ is 0.08 smaller than that of Scott et al. (2014) with the same solar model assumption. If we repeat the Scott et al. EW analysis with their $\log (g f)$ values we recover their mean abundance. However, for their lines our transition probabilities are 0.02 dex larger on average than they used, and thus their revised abundance
Table 7

Sixty-eight Lines of Ionized Vanadium in HD 84937

\begin{tabular}{lcccc}
\hline \hline $\begin{array}{l}\text { Wavelength in Air } \\
(\AA)\end{array}$ & $\begin{array}{c}\text { Excitation Energy } \\
(\mathrm{eV})\end{array}$ & $\log _{10}(g f)$ & $\log _{10}(\varepsilon)$ & HFS $^{\mathrm{a}}$ \\
\hline 2677.796 & 0.004 & -0.76 & 1.85 & no \\
2679.316 & 0.026 & -0.63 & 1.92 & yes \\
2687.951 & 0.042 & -0.08 & 1.85 & yes \\
2688.708 & 0.042 & -0.98 & 1.85 & yes \\
2690.241 & 0.026 & -0.76 & 1.93 & no \\
\hline
\end{tabular}

Notes.

a This column denotes whether or not hyperfine substructure has been included in the abundance calculation.

(This table is available in its entirety in a machine-readable form in the online journal. A portion is shown here for guidance regarding its form and content.)

would become 4.01 with application of our $\log (g f)$ values. Our synthetic spectrum analysis of the Scott et al. lines yields $\langle\log \varepsilon(\mathrm{V})\rangle=3.97$, another decrease of 0.04 dex. We suspect that the synthetic spectrum approach may account better for blending transitions in the crowded near-UV spectral region where the V II lines arise. Finally, we note that Scott et al. (2014) recommend a solar-system meteoritic abundance of $\langle\log \varepsilon(\mathrm{V})\rangle=3.96 \pm 0.02$, which is in excellent accord with our photospheric $\mathrm{V}$ abundance.

\section{THE VANADIUM ABUNDANCE OF METAL-POOR STAR HD 84937}

Determining vanadium abundances in low metallicity stars from new V II lab data continues our efforts to apply improved basic lab data to Fe-group elements whose nucleosynthesis and Galactic evolution can be predicted theoretically (e.g., Kobayashi et al. 2006, and references therein). Concerns exist about the reliability of Fe-group abundances at low metallicity, including the need to gather spectra of high quality UV and near-UV wavelengths, and the need to explore the limitations of standard analytical assumptions (e.g. LTE, plane-parallel geometry) used in deriving abundances. Here we see what can be understood from application of traditional abundance techniques to this new large set of accurate V II line data for one well-studied star.

HD 84937 is a metal-poor main-sequence turnoff $\operatorname{star}\left(T_{\text {eff }}=\right.$ $6300 \mathrm{~K}, \log g=4.0,[\mathrm{Fe} / \mathrm{H}]=-2.15$, and $\left.v_{\mathrm{t}}=1.5 \mathrm{~km} \mathrm{~s}^{-1}\right)$. Inspection of its near-UV spectrum reveals many promising V II transitions, since contamination from other species decreases greatly and many V II lines that are completely saturated in the solar photosphere weaken enough to be useful abundance indicators. We derive the $\mathrm{V}$ abundance in HD 84937 in similar fashion to our previous Fe-group abundance studies.

Our data set for HD 84937 consists of an optical ESO VLT UVES spectrum and an HST/STIS UV high-resolution spectrum $^{12}$. Lawler et al. (2013) describe these spectra in detail. The availability of a UV spectrum for this star causes us to repeat the transition candidate selection process from the beginning, which results in nearly 75 possible V II lines for analysis. Lineby-line abundance derivation via spectrum syntheses is done as described in Lawler et al. (2013). This yields a mean abundance in $\mathrm{HD} 84937$ of $\langle\log \varepsilon(\mathrm{V})\rangle=1.871 \pm 0.009$, with $\sigma=0.075$ from 68 lines. Individual abundances derived from V II lines in HD 84937 are listed in Table 7 and displayed in the lower panel of Figure 7 . Then with the mean solar abundance derived

\footnotetext{
12 Obtained from the $H S T$ archive; the spectrum was gathered originally under
} proposal \#7402 (PI: R. C. Peterson). 
in Section 6, we compute a relative $\mathrm{V}$ abundance of $\langle[\mathrm{V} / \mathrm{H}]\rangle=$ $1.87-3.95=-2.08$. There are six V II lines in common between our analyses of the solar photosphere (Table 6) and HD 84937 (Table 7). From just those lines, we derive $\langle[\mathrm{V} / \mathrm{H}]\rangle=-2.08 \pm$ $0.03(\sigma=0.08)$, in excellent agreement with the value computed from the complete line sets for both stars.

Following the discussion of Lawler et al. (2013), we estimate that internal line-to-line scatter uncertainties are $\leqslant 0.04 \mathrm{dex}$. For external error estimates, we derive abundances of 14 typical V II lines with model atmosphere parameters varied in accord with the HD 84937 uncertainties. If $T_{\text {eff }}$ is increased by $150 \mathrm{~K}$, then on average $\Delta(\log \varepsilon) \approx+0.09$; if $\log g$ is increased by 0.3 , then $\Delta(\log \varepsilon) \approx+0.08$; if the metallicity is decreased to $[\mathrm{Fe} / \mathrm{H}]=$ -2.45 , then $\Delta(\log \varepsilon) \approx 0.00$ (unchanged); and if $v_{\mathrm{t}}$ is decreased to $1.25 \mathrm{~km} \mathrm{~s}^{-1}$ then $\Delta(\log \varepsilon) \approx+0.00$ to +0.08 , depending on the strength of the measured transition. However, we note that the response of V II transitions to changes in model atmospheric parameters is very similar to other ions of the Fe-group, which have similar Saha ionization properties. Thus [V/Ti] or $[\mathrm{V} / \mathrm{Fe}]$ relative abundance ratios are mostly insensitive to model parameter variations. We reserve comment on the statistical equilibrium of vanadium in HD 84937 until completion of our study of V I transitions in the Sun and this star (Lawler et al. 2014).

\section{IMPLICATIONS FOR Fe-GROUP NUCLEOSYNTHESIS}

Our group has recently been examining the Fe-peak abundances in both the Sun and selected metal-poor stars. The Fepeak elements were synthesized in Type II supernovae ( $\mathrm{SNe}$ ) early in the history of the Galaxy. As described in the previous section, our new mean photospheric abundance for HD 84937 is $[\mathrm{V} / \mathrm{H}]=2.08$ (based upon $\log \varepsilon(\mathrm{V})=1.87$ for HD 84937 and $\log \varepsilon(\mathrm{V})=3.95$ for the Sun). Lawler et al. (2013) had previously found that $[\mathrm{Fe} / \mathrm{H}]=-2.32$ in $\mathrm{HD} 84937$, leading to a newly determined value of $[\mathrm{V} / \mathrm{Fe}]=0.24$ for this metal-poor star. Vanadium is an odd- $Z$ element, and as such it has a lower photospheric abundance than the nearby (even- $Z$ ) Fe-peak element $\mathrm{Ti}$, even though it is made in a similar manner (see discussion in Lawler et al. 2013). For HD 84937, we now have new precise values for $[\mathrm{Ti} / \mathrm{Fe}]=0.47$ (Lawler et al. 2013, Wood et al. 2013), $[\mathrm{Ni} / \mathrm{Fe}]=-0.07($ Wood et al. 2014) and $[\mathrm{V} / \mathrm{Fe}]=0.24$ (this paper). These new abundance ratios can provide constraints on inputs for $\mathrm{SNe}$ models. The Fe-peak elements are synthesized in either complete (e.g., Ni) or incomplete (e.g., Ti and V) silicon burning during the (core collapse) SN phase. Thus, the abundances are a direct indication of such parameters as the SN energy and where the mass cut, the boundary above which matter is ejected in the explosion, is located (see Nakamura et al. 1999). Previously, Lawler et al. (2013) suggested that the precise value of the Ti abundance in HD 84937 was higher than various $\mathrm{SNe}$ model predictions. One can employ the new V abundance value reported herein to determine $[\mathrm{V} / \mathrm{Ti}]=-0.23$ in this one metal-poor star. The only major stable isotope of vanadium is ${ }^{51} \mathrm{~V}$ (see footnote 8), and therefore model predictions (see, e.g., Thielemann et al. 1996) would need to compare mass production of isotopic abundances 51 and 48 (the main isotope for Ti) in, for example, a typical $15 M_{\odot}$ model.

There have not been extensive listings of data for $\mathrm{V}$, and in particular V II, in the literature. However, previous studies (e.g., Lai et al. 2008, Henry et al. 2010) have suggested that [V/Fe] is solar (i.e., remains flat) over a wide range of metallicity. Our new value for $\mathrm{HD} 84937$ of $[\mathrm{V} / \mathrm{Fe}]=0.24$ at $[\mathrm{Fe} / \mathrm{H}]=-2.32$ is slightly elevated, and suggests that $[\mathrm{V} / \mathrm{Fe}]$ might rise at lower metallicity. We caution that there is some uncertainty in the $\mathrm{Fe}$

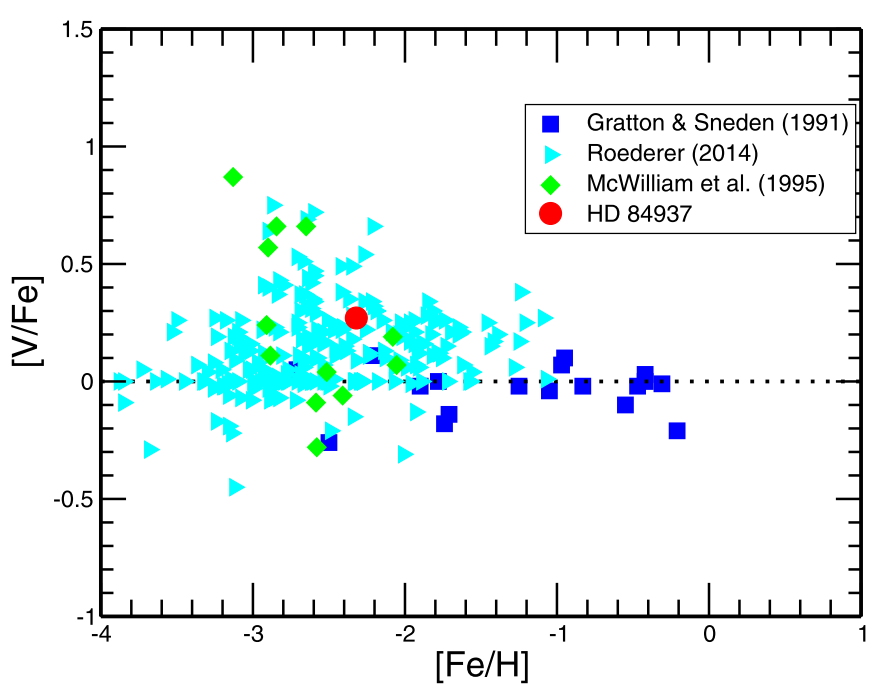

Figure 8. $[\mathrm{V} / \mathrm{Fe}]$ values as a function of metallicity, showing a slight rise and large scatter for $[\mathrm{Fe} / \mathrm{H}]<-1$. As indicated in the figure legend, the data sources are McWilliam et al. (1995a) (green diamonds), Gratton \& Sneden (1991) (blue squares), and I. U. Roederer (2014, private communication) (cyan triangles), with the HD 84937 result presented in the paper indicated by the red circle.

(A color version of this figure is available in the online journal.)

abundance, which could then lead to uncertainty in the relative [V/Fe] abundance ratio. McWilliam et al. (1995a) had found a few stars with very high $[\mathrm{V} / \mathrm{Fe}]$ values at low metallicities (see also Johnson 2002). Recently, I. U. Roederer (2014, private communication) has assembled a large data base of elements in metal-poor stars, and in Figure 8 we plot those new data for $[\mathrm{V} / \mathrm{Fe}$ ] along with that of McWilliam et al. (1995a) and Gratton \& Sneden (1991). There does indeed appear to be a rise in $[\mathrm{V} / \mathrm{Fe}]$, with large scatter, at very low metallicities. Our new value for HD 84937, indicated by a filled red circle, is consistent with this increasing scatter in $[\mathrm{V} / \mathrm{Fe}]$ that starts to occur below a metallicity of approximately $[\mathrm{Fe} / \mathrm{H}]=-1$. Clearly, additional studies - abundance analyses of additional stars are planned in the near future-will be required to identify any $\mathrm{V}$ abundance trends at low metallicities.

\section{SUMMARY}

We report 203 new experimental transition probabilities in V II from a combination of branching fractions measured using FTS and echelle spectra and new LIF radiative lifetimes. Generally good agreement is found with previously reported V II transition probabilities. The use of two spectrometers with independent radiometric calibration methods leads to a reduction in systematic uncertainties and allows for a more thorough examination of optical depth effects. The FTS spectra also yield new measurements for V II HFS constants of selected levels. The new V II transition probabilities and HFS data are used to re-determine the vanadium abundance of the Sun and metal-poor star HD 84937 using lines covering a range of wavelength, E.P., and $\log (g f)$ values to search for non-LTE effects. Our new solar photospheric vanadium abundance, log $\varepsilon(\mathrm{V})=3.95$, is slightly lower than previous results, but shows excellent agreement with the solar-system meteoritic value. In HD 84937, we derive $[\mathrm{V} / \mathrm{H}]=-2.08$, yielding a value of $[\mathrm{V} / \mathrm{Fe}]=0.24$ for this star, which is consistent with a rise in $[\mathrm{V} / \mathrm{Fe}]$ at low metallicity subject to uncertainty in the $\mathrm{Fe}$ abundance. 
The authors acknowledge the contribution of N. Brewer on data analysis for this project. This work is supported in part by NASA grant NNX10AN93G (J.E.L.) and NSF grant AST1211585 (C.S.). The authors thank Ian Roederer for making available his abundance data on metal-poor stars.

\section{REFERENCES}

Adams, D. L., \& Whaling, W. 1981, JOSA, 71, 1036

Asplund, M. 2005, ARA\&A, 43, 481

Armstrong, N. M. R., Rosner, S. D., \& Holt, R. A. 2011, PhyS, 84, 055301

Arvidsson, K. 2003, Master's thesis, Lund Observatory, Lund Univ., Sweden

Barklem, P. S., Christlieb, N., Beers, T. C., et al. 2005, A\&A, 439, 129

Beck, D., \& Abdalmoneam, M. 2014, BAAS, 59, 8 (http://meetings.aps.org/link/ BAPS.2014.DAMOP.D1.40)

Biémont, E., Grevesse, N., Faires, L. M., et al. 1989, A\&A, 209, 391

Bonifacio, P., Spite, M., Cayrel, R., et al. 2009, A\&A, 501, 519

Brault, J. W. 1976, JOSA, 66, 1081

Cayrel, R., Depange, E., Spite, M., et al. 2004, A\&A, 416, 1117

Cowan, J. J., Sneden, C., Spite, M., et al. 2002, ApJ, 572, 861

Danzmann, K., \& Kock, M. 1982, JOSA, 72, 1556

Delbouille, L., Roland, G., \& Neven, L. 1973, Photometric Atlas of the Solar Spectrum from $\lambda 3000$ to $\lambda 10000$ (Liège: Inst. d'Ap., Univ. de Liège)

Den Hartog, E. A., Lawler, J. E., Sobeck, J. S., Sneden, C., \& Cowan, J. J. 2011, ApJS, 194, 35

Den Hartog, E. A., Lawler, J. E., \& Wood, M. P. 2014, ApJS, in press

Gratton, R. G., \& Sneden, C. 1991, A\&A, 541, 501

Hashiguchi, S., \& Hasikuni, M. 1985, JPSJ, 54, 1290

Henry, R. B. C., Cowan, J. J., \& Sobeck, J. 2010, ApJ, 709, 715

Holweger, H., \& Müller, E. A. 1974, SoPh, 39, 19

Johnson, J. A. 2002, ApJS, 139, 219

Karamatskos, N., Michalak, R., Zimmermann, P., Kroll, S., \& Kock, M. 1986, ZPhyD, 3, 391

Kramida, A., Ralchenko, Y., Reader, J., \& NIST ASD Team 2013, NIST Atomic Spectra Database (version 5.1; Gaithersburg, MD: US NIST)

Kobayashi, C., Umeda, H., Nomoto, K., Tominaga, N., \& Ohkubo, T. 2006, ApJ, 653,1145

Kurucz, R. L. 2011, CaJPh, 89, 417
Lai, D. K., Bolte, M., Johnson, J. A., et al. 2008, ApJ, 681, 1524

Lawler, J. E., Bonvallet, G., \& Sneden, C. 2001a, ApJ, 556, 452

Lawler, J. E., Guzman, A., Wood, M. P., Sneden, C., \& Cowan, J. J. 2013, ApJS, 205,11

Lawler, J. E., Wickliffe, M. E., Den Hartog, E. A, \& Sneden, C. 2001b, ApJ, 563, 1075

Lawler, J. E., Wood, M. P., Den Hartog, E. A., et al. 2014, ApJS, submitted

McWilliam, A. 1997, ARA\&A, 35, 503

McWilliam, A., Preston, G. W., Sneden, C., \& Searle, L. 1995a, AJ, 109,2757

McWilliam, A., Preston, G. W., Sneden, C., \& Shectman, S. 1995b, AJ, 109,2736

Moore, C. E., Minnaert, M. G. J., \& Houtgast, J. 1966, The Solar Spectrum $2935 \AA$ to $8770 \AA$ (NBS Monograph Vol. 61; Washington, DC: US GPO)

Nakamura, T., Umeda, H., Nomoto, K., Thielemann, F.-K., \& Burrows, A. 1999, ApJ, 517, 193

Peck, E. R., \& Reeder, K. 1972, JOSA, 62, 958

Roberts, J. R., Andersen, T., \& Sørenson, G. 1973, ApJ, 181, 567

Roederer, I. U. 2009, AJ, 137, 272

Scott, P., Asplund, M., Grevesse, N., Bergemann, M., \& Jacques Sauval, A. 2014, arXiv: 1405.0287

Sneden, C. 1973, ApJ, 184, 839

Sneden, C., Cowan, J. J., Lawler, J. E., et al. 2003, ApJ, 591, 936

Sneden, C., Lawler, J. E., Cowan, J. J., Ivans, I. I., \& Den Hartog, E. A. 2009, ApJS, 182, 80

Sobeck, J. S., Lawler, J. E., \& Sneden, C. 2007, ApJ, 667, 1267

Suda, T., Yamada, S., Katsuna, Y., et al. 2011, MNRAS, 412, 843

Thielemann, F.-K., Nomoto, K., \& Hashimoto, M. A. 1996, ApJ, 460, 408

Thorne, A. P., Pickering, J. C., \& Semeniuk, J. I. 2013, ApJS, 207, 13

Westin, J., Sneden, C., Gustafsson, B., \& Cowan, J. J. 2000, ApJ, 530, 783

Whaling, W., Carle, M. T., \& Pitt, M. L. 1993, JQSRT, 50, 7

Wickliffe, M. E., Lawler, J. E., \& Nave, G. 2000, JQSRT, 66, 363

Wood, M. P., \& Lawler, J. E. 2012, ApOpt, 51, 8407

Wood, M. P., Lawler, J. E., Sneden, C., \& Cowan, J. J. 2013, ApJS, 208, 27

Wood, M. P., Lawler, J. E., Sneden, C., \& Cowan, J. J. 2014, ApJS, 211, 20

Woodgate, G. K. 1980, Elementary Atomic Structure (Oxford: Oxford Univ. Press)

Wujec, T., \& Musielok, J. 1986, JQSRT, 36, 7

Xu, H., Jiang, Z., \& Lundberg, H. 2006, JOSA B, 23, 2597

Yong, D., Norris, J. E., Bessell, M. S., et al. 2013, ApJ, 762, 26 